\title{
2012 National Child Poverty Rate Stagnates at 22.6 Percent
}

\section{New Hampshire Child Poverty Jumps 30 Percent Since 2011}

Marybeth J. Mattingly, Jessica A. Carson, and Andrew Schaefer

$\mathrm{S}$ tubbornly high poverty rates in the wake of the Great Recession suggest we have not yet "turned the corner" three years after its official end. In 2012, the child poverty rate was 22.6 percent, not statistically different from 2011, but roughly 4.6 percentage points higher than in 2007 (see Table 1). ${ }^{1}$ There is wide variation in child poverty rates by state and region, with the highest rates in the South and the lowest rates in the Northeast. The largest regional place increase in child poverty both from 2011 to 2012 and 2007 to 2012 was in central cities in the West (up 0.9 and 6.3 percentage points, respectively). See Figure 1.

Children under age 18 are least often poor in suburban America, where 2012 rates are estimated at 17.2 percent nationally, as compared to 26.2 percent in rural places and 29.7 percent in central cities. However, in absolute numbers, many more poor children live in the central cities and suburbs, as compared to rural places.

Child poverty increased the most in New Hampshire and Mississippi (by 3.6 and 2.9 percentage points, respectively, see Table 1). The significant increase in New Hampshire is particularly surprising given that it has consistently had the lowest rate in the nation. ${ }^{2}$ In 2011, New Hampshire's child poverty was lower than all other states and Washington, DC. However, in 2012, New Hampshire's rate was lower than only thirty-five states. ${ }^{3}$

Within New Hampshire, child poverty was stable in the suburbs but increased in rural places and central cities. Child poverty also increased in Nevada,

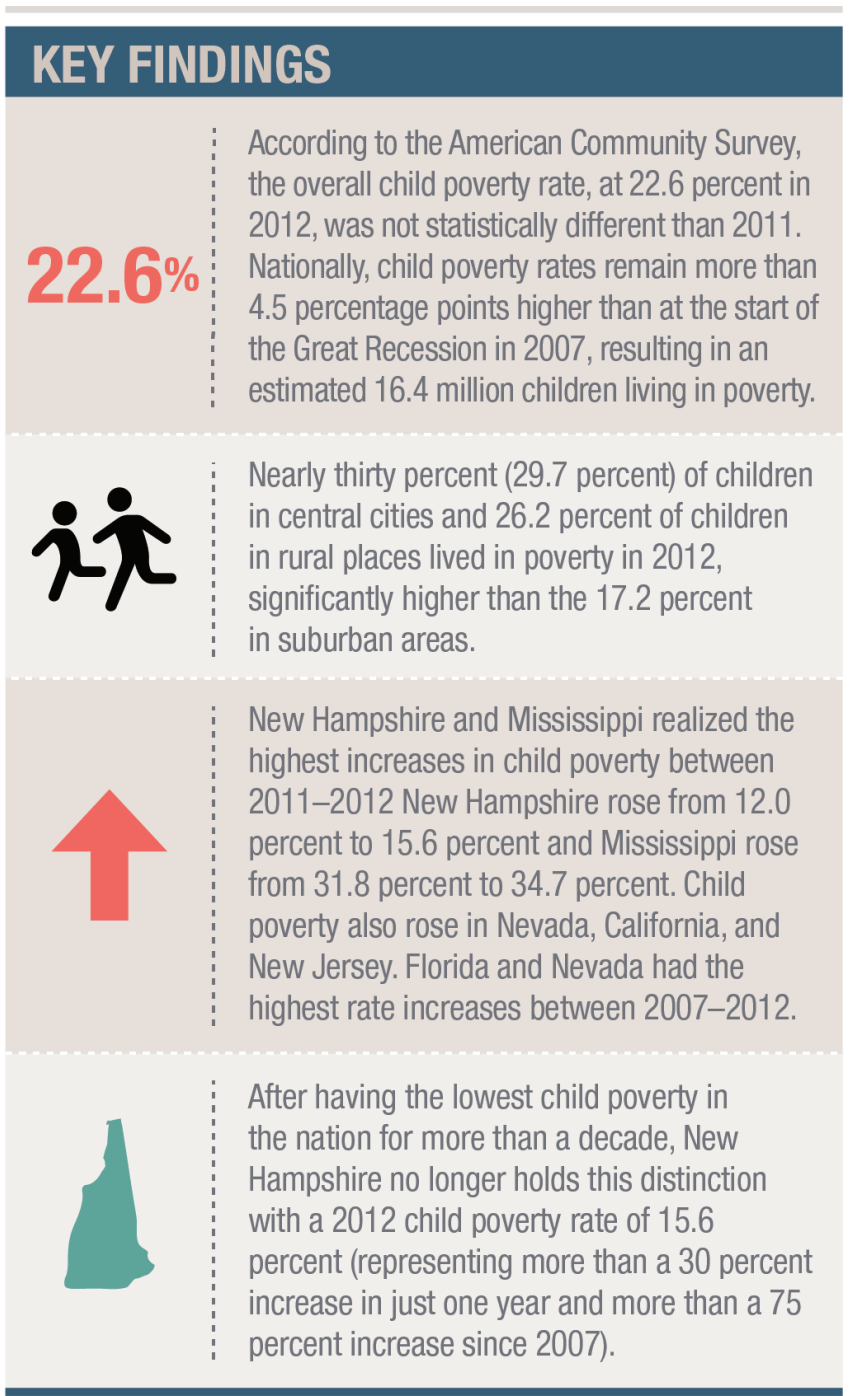




\section{Box 1: Poverty Measurement}

Documenting trends using official poverty rates,

as in this brief, enables comparisons of child

poverty across places, and offers a method for

consistently comparing the adequacy of families'

incomes for meeting children's needs. However,

the official poverty measure has important limi-

tations: for example, the measure is sometimes

critiqued as dated, relying on a formula estab-

lished about fifty years ago to calculate annual

poverty rates. It also does not consider how

work-related expenses (such as child care), in-

kind assistance (for example, food stamps, or the

Supplemental Nutrition Assistance Program),

medical costs (such as insurance premiums),

post-tax transfers (such as the Earned Income

Tax Credit), or geographic differences in the cost

of living impact families' resources and expenses.

An alternate measure, the Research Supplemental

Poverty Measure, has been offered by the Census

Bureau as a potentially more nuanced alternative

that considers the aforementioned resources and

expenses when calculating poverty rates. As more

data become available on this measure, we can

compare these newly estimated poverty rates to

official poverty measurements, and consider how

these alternate calculations influence our under-

standing of the landscape of poverty.

California, and New Jersey. In contrast, child poverty fell in four states: Iowa, Illinois, Texas, and Minnesota, but remains 2.3 to 4.0 percentage points higher than in 2007 in these states.

\section{Young Child Poverty}

Poverty among young children is of particular concern because children under age 6 are the age group that typically has the highest poverty rate and because young children who are poor experience the most adverse outcomes later in life. ${ }^{4}$

In 2012, an estimated 25.5 percent of young children in America lived in families with incomes below the federal poverty threshold (see Table 2). Young child poverty rose slightly in the West (by 0.5 percentage point) and Northeast ( 0.4 percentage point) and fell slightly in the Midwest (by 0.7 percentage point) between 2011 and 2012. Young child poverty is highest in the South and lowest in the Northeast. Young child poverty is consistently higher in central cities and rural places than in the suburbs.

\section{Conclusion}

These new poverty estimates released on September 19, 2013 suggest that child poverty plateaued in the aftermath of the Great Recession, but there is no evidence of any reduction in child poverty even as we enter the fourth year of "recovery." Modest improvements are evident in some places, but in others rates have increased, raising concerns about the well-being of America's children. This is particularly evident in our own state, New Hampshire, where the child poverty rate rose from 12.0 to 15.6 percent. This means that, on average, for every twenty-eight children in New Hampshire, there was an additional child in poverty in 2012 as compared to 2011.

Given the continuing high levels of child poverty, it is imperative to consider the role of the safety net in protecting America's most vulnerable. Programs like the Earned Income Tax Credit (EITC), the Supplemental Nutrition Assistance Program (SNAP), and Temporary Assistance to Needy Families (TANF) provide critical support for poor children. For example, SNAP has been shown to improve young children's health and reduce developmental delays. ${ }^{5}$ The EITC has been shown to improve the health of infants, and young children whose families receive the credit do better in school and earn more as adults. ${ }^{6}$

\section{D a t a}

While the Community Population Survey (CPS) data are useful for providing a snapshot of official poverty across the nation, the larger sample size of the American Community Survey (ACS), which samples three million addresses, versus 100,000 addresses in the CPS, is better suited for nuanced analyses of poverty, particularly by place and demographic subgroups. In this brief, we use the ACS data released on September 19, 2013, to address patterns of poverty among children, exploring variations by state, region, and place type. ${ }^{7}$ 


\section{FIGURE 1. PERCENT OF CHILDREN IN POVERTY, 2012}

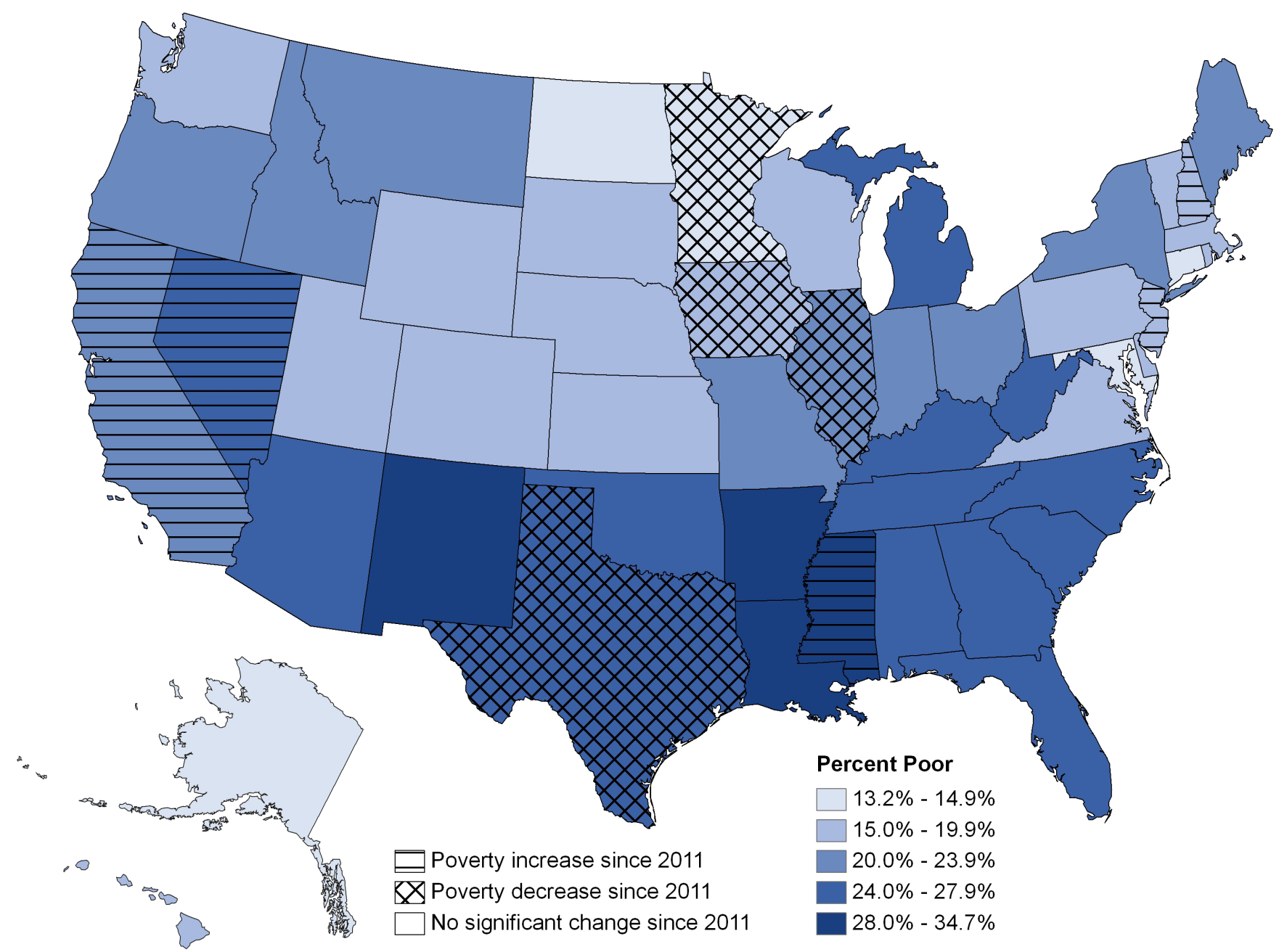

Source: Carsey Institute analysis of 2011 \& 2012 ACS, U.S. Census Bureau

This analysis is based on estimates from the 2007, 2011, and 2012 American Community Survey. ${ }^{8}$ Tables were produced by aggregating information from detailed tables available on American FactFinder (http:// factfinder2.census.gov/faces/nav/jsf/pages/index.xhtml). These estimates give perspective on child poverty, but they are based on survey data, so caution must be exercised in comparing across years or places. ${ }^{9}$ All differences highlighted in this brief and bolded in the tables are statistically significant $(p<0.05)$.
Box 2: Definition of the Terms Rural, Suburban, and Central City

Data for this brief are derived from the American Community Survey, which locates each address as being within one of several geographic components. As used here, "central city" designates households in the principal city of a given metropolitan statistical area, and "suburban" includes those in metropolitan areas, but not within the principal city of that area. "Rural" consists of the addresses that are not within a metropolitan area. 
TABLE 1. CHILD POVERTY BY STATE AND PLACE TYPE IN 2012

\begin{tabular}{|c|c|c|c|c|c|c|c|c|}
\hline & \multicolumn{4}{|c|}{ ALL PLACES } & \multicolumn{4}{|c|}{ RURAL } \\
\hline & $\begin{array}{l}\text { Number Below } \\
\text { Poverty }\end{array}$ & $\begin{array}{l}\text { Percent Below } \\
\text { Poverty }\end{array}$ & $\begin{array}{l}\text { Percentage } \\
\text { Point Change } \\
\text { Since } 2011\end{array}$ & $\begin{array}{l}\text { Percentage } \\
\text { Point Change } \\
\text { Since } 2007\end{array}$ & $\begin{array}{l}\text { Number Below } \\
\text { Poverty }\end{array}$ & $\begin{array}{l}\text { Percent Below } \\
\text { Poverty }\end{array}$ & $\begin{array}{l}\text { Percentage } \\
\text { Point Change } \\
\text { Since } 2011\end{array}$ & $\begin{array}{l}\text { Percentage } \\
\text { Point Change } \\
\text { Since } 2007\end{array}$ \\
\hline United States & $16,400,000$ & 22.58 & 0.08 & 4.56 & $2,925,830$ & 26.19 & -0.09 & 4.26 \\
\hline Northeast & $2,287,329$ & 19.16 & 0.38 & 3.49 & 221,157 & 20.74 & 0.67 & 4.35 \\
\hline Midwest & $3,261,456$ & 20.95 & -0.43 & 4.25 & 723,741 & 21.27 & -0.72 & 3.86 \\
\hline South & $6,876,496$ & 25.01 & -0.07 & 4.39 & $1,587,451$ & 31.37 & 0.28 & 4.59 \\
\hline West & $3,971,582$ & 22.57 & 0.51 & 5.68 & 393,481 & 24.00 & -0.49 & 3.83 \\
\hline Alabama & 305,610 & 27.48 & -0.12 & 3.17 & 100,477 & 32.48 & 1.34 & 5.15 \\
\hline Alaska & 25,700 & 13.91 & -0.64 & 2.41 & 9,510 & 20.63 & 0.28 & 1.08 \\
\hline Arizona & 429,486 & 26.97 & -0.26 & 6.80 & 41,355 & 33.76 & -4.52 & 2.70 \\
\hline Arkansas & 199,617 & 28.52 & 0.43 & 2.74 & 89,061 & 33.82 & 1.61 & 4.95 \\
\hline California & $2,167,372$ & 23.81 & 0.97 & 6.55 & 41,493 & 25.33 & -0.23 & 6.91 \\
\hline Colorado & 223,970 & 18.47 & 0.58 & 2.15 & 31,895 & 21.91 & 1.17 & 3.15 \\
\hline Connecticut & 116,536 & 14.85 & -0.10 & 3.78 & 6,731 & 10.69 & 0.61 & 3.17 \\
\hline Delaware & 34,875 & 17.36 & -0.10 & 2.69 & 11,262 & 28.65 & 9.02 & 15.02 \\
\hline District of Columbia & 28,623 & 26.53 & -3.80 & 3.82 & N/A & N/A & $\mathrm{N} / \mathrm{A}$ & N/A \\
\hline Florida & $1,000,736$ & 25.39 & 0.48 & 8.32 & 71,107 & 35.03 & 0.51 & 13.52 \\
\hline Georgia & 672,040 & 27.24 & 0.89 & 7.54 & 150,840 & 35.30 & 1.18 & 9.43 \\
\hline Hawaii & 51,233 & 17.11 & 0.12 & 7.36 & 19,467 & 21.34 & -4.07 & 10.03 \\
\hline Idaho & 86,532 & 20.66 & 0.23 & 4.79 & 31,862 & 23.33 & 2.03 & 5.65 \\
\hline Illinois & 624,272 & 20.67 & -0.88 & 4.04 & 72,674 & 20.83 & -0.08 & 3.41 \\
\hline Indiana & 349,524 & 22.37 & -0.67 & 5.11 & 75,209 & 22.59 & -1.14 & 4.65 \\
\hline lowa & 112,573 & 15.85 & -1.44 & 2.26 & 52,542 & 17.63 & 0.43 & 2.90 \\
\hline Kansas & 135,006 & 19.03 & 0.22 & 4.45 & 46,688 & 21.87 & 0.94 & 5.01 \\
\hline Kentucky & 263,819 & 26.51 & -0.92 & 2.59 & 129,995 & 32.71 & -0.31 & 3.42 \\
\hline Louisiana & 310,053 & 28.14 & -0.64 & 1.39 & 86,537 & 30.59 & -1.43 & -3.26 \\
\hline Maine & 54,065 & 20.87 & 2.03 & 5.51 & 24,826 & 23.69 & 2.29 & 5.24 \\
\hline Maryland & 183,044 & 13.82 & 0.31 & 3.34 & 9,290 & 13.75 & -3.59 & 2.73 \\
\hline Massachusetts & 213,206 & 15.41 & 0.18 & 2.48 & $N / A$ & $N / A$ & $\mathrm{~N} / \mathrm{A}$ & $\mathrm{N} / \mathrm{A}$ \\
\hline Michigan & 554,153 & 24.87 & 0.04 & 5.43 & 90,013 & 23.84 & -1.66 & 4.23 \\
\hline Minnesota & 183,763 & 14.57 & -0.84 & 2.57 & 50,893 & 16.99 & -0.89 & 3.62 \\
\hline Mississippi & 255,839 & 34.69 & 2.86 & 5.38 & 161,171 & 40.50 & 3.60 & 5.37 \\
\hline Missouri & 310,229 & 22.61 & 0.52 & 4.89 & 91,419 & 27.10 & -0.40 & 5.54 \\
\hline Montana & 43,733 & 20.30 & 0.60 & 1.97 & 29,520 & 21.00 & 0.38 & 0.63 \\
\hline Nebraska & 80,839 & 17.86 & -0.28 & 2.93 & 30,988 & 17.48 & -1.02 & 1.41 \\
\hline Nevada & 156,523 & 23.95 & 1.86 & 8.67 & 10,622 & 21.43 & 0.63 & 7.51 \\
\hline New Hampshire & 42,069 & 15.57 & 3.61 & 6.82 & 15,599 & 18.35 & 4.81 & 9.52 \\
\hline New Jersey & 310,226 & 15.45 & 0.78 & 3.87 & N/A & N/A & $\mathrm{N} / \mathrm{A}$ & $\mathrm{N} / \mathrm{A}$ \\
\hline New Mexico & 149,404 & 29.33 & -1.38 & 3.87 & 52,698 & 30.41 & -2.41 & 2.63 \\
\hline New York & 958,610 & 22.83 & 0.26 & 3.41 & 74,215 & 23.28 & -1.57 & 3.84 \\
\hline North Carolina & 586,104 & 26.00 & 0.41 & 6.46 & 197,470 & 31.38 & 0.41 & 7.03 \\
\hline North Dakota & 19,841 & 13.15 & -1.49 & -0.27 & 12,219 & 15.34 & -2.06 & 0.04 \\
\hline Ohio & 620,921 & 23.77 & -0.42 & 5.25 & 123,195 & 24.25 & -0.92 & 5.64 \\
\hline Oklahoma & 221,623 & 24.12 & 0.68 & 1.62 & 83,994 & 26.80 & 0.77 & 1.60 \\
\hline Oregon & 195,093 & 23.03 & -0.61 & 6.10 & 47,357 & 26.55 & -1.09 & 5.41 \\
\hline Pennsylvania & 532,166 & 19.74 & 0.19 & 3.43 & 83,058 & 20.72 & 0.77 & 3.90 \\
\hline Rhode Island & 41,635 & 19.45 & -2.42 & 1.98 & $N / A$ & $\mathrm{~N} / \mathrm{A}$ & $\mathrm{N} / \mathrm{A}$ & $\mathrm{N} / \mathrm{A}$ \\
\hline South Carolina & 287,664 & 26.92 & -0.87 & 6.00 & 81,813 & 34.07 & -0.16 & 7.99 \\
\hline South Dakota & 34,901 & 17.49 & -0.67 & 0.70 & 21,569 & 20.64 & -1.96 & -0.63 \\
\hline Tennessee & 379,319 & 25.83 & -0.43 & 2.84 & 103,070 & 28.24 & -2.10 & 1.86 \\
\hline Texas & $1,776,664$ & 25.76 & -0.86 & 2.57 & 210,491 & 28.45 & -0.37 & 1.75 \\
\hline Utah & 131,915 & 15.12 & -0.75 & 4.13 & 13,227 & 13.59 & -2.58 & -3.71 \\
\hline Vermont & 18,816 & 15.47 & 0.58 & 3.12 & 14,256 & 17.83 & 1.91 & 2.95 \\
\hline Virginia & 278,899 & 15.25 & -0.06 & 2.23 & 54,508 & 24.53 & -2.77 & 5.15 \\
\hline Washington & 288,147 & 18.52 & 0.21 & 3.49 & 43,460 & 24.83 & 1.45 & 4.75 \\
\hline West Virginia & 91,967 & 24.59 & -1.25 & 1.80 & 46,365 & 28.51 & 1.03 & 2.22 \\
\hline Wisconsin & 235,434 & 18.24 & 0.00 & 3.82 & 56,332 & 17.28 & -0.96 & 2.98 \\
\hline Wyoming & 22,474 & 16.91 & 1.29 & 5.29 & 16,212 & 17.24 & 1.32 & 3.55 \\
\hline
\end{tabular}

Note: Percentage point changes are based on unrounded poverty percentages and may differ slightly from those that 


\section{TABLE 1. CHILD POVERTY BY STATE AND PLACE TYPE IN 2012, CONTINUED}

\begin{tabular}{|c|c|c|c|c|c|c|c|c|}
\hline & \multicolumn{4}{|c|}{ SUBURBAN } & \multicolumn{4}{|c|}{ CENTRAL CITY } \\
\hline & $\begin{array}{l}\text { Number Below } \\
\text { Poverty }\end{array}$ & $\begin{array}{c}\text { Percent Below } \\
\text { Poverty }\end{array}$ & $\begin{array}{l}\text { Percentage } \\
\text { Point Change } \\
\text { Since } 2011\end{array}$ & $\begin{array}{c}\text { Percentage } \\
\text { Point Change } \\
\text { Since } 2007\end{array}$ & $\begin{array}{l}\text { Number Below } \\
\text { Poverty }\end{array}$ & $\begin{array}{l}\text { Percent Below } \\
\text { Poverty }\end{array}$ & $\begin{array}{l}\text { Percentage } \\
\text { Point Change } \\
\text { Since } 2011\end{array}$ & $\begin{array}{c}\text { Percentage } \\
\text { Point Change } \\
\text { Since } 2007\end{array}$ \\
\hline United States & $6,549,634$ & 17.19 & 0.05 & 4.33 & $6,921,399$ & 29.67 & 0.17 & 5.02 \\
\hline Northeast & 906,338 & 12.35 & 0.37 & 3.14 & $1,159,834$ & 32.84 & 0.00 & 4.25 \\
\hline Midwest & $1,156,813$ & 14.77 & -0.40 & 3.79 & $1,380,902$ & 31.88 & -0.38 & 5.33 \\
\hline South & $2,786,360$ & 19.59 & -0.15 & 4.48 & $2,502,685$ & 30.48 & -0.11 & 4.10 \\
\hline West & $1,700,123$ & 19.53 & 0.42 & 5.41 & $1,877,978$ & 25.89 & 0.85 & 6.34 \\
\hline Alabama & 107,254 & 21.01 & -0.65 & 0.64 & 97,879 & 33.50 & -1.01 & 5.22 \\
\hline Alaska & 3,494 & 8.29 & -5.24 & -0.65 & 10,303 & 12.52 & -0.28 & 3.14 \\
\hline Arizona & 121,049 & 18.14 & -4.19 & 2.38 & 267,082 & 33.27 & 3.71 & 10.99 \\
\hline Arkansas & 47,206 & 20.29 & -2.13 & -0.70 & 63,350 & 31.07 & 1.78 & 3.99 \\
\hline California & $1,038,564$ & 22.23 & 1.60 & 7.03 & $1,087,315$ & 25.47 & 0.33 & 5.97 \\
\hline Colorado & 81,811 & 13.54 & 0.38 & 2.28 & 110,264 & 23.80 & 0.56 & 1.18 \\
\hline Connecticut & 50,972 & 10.25 & 0.32 & 2.62 & 58,833 & 26.18 & -1.53 & 5.85 \\
\hline Delaware & 15,334 & 11.24 & -2.80 & -0.23 & 8,279 & 33.00 & 0.34 & -2.45 \\
\hline District of Columbia & N/A & $\mathrm{N} / \mathrm{A}$ & $\mathrm{N} / \mathrm{A}$ & N/A & 28,623 & 26.53 & -3.80 & 3.82 \\
\hline Florida & 625,637 & 23.25 & 1.25 & 8.08 & 303,992 & 29.02 & -1.57 & 7.62 \\
\hline Georgia & 371,229 & 22.43 & 0.02 & 7.45 & 149,971 & 38.94 & 4.06 & 6.37 \\
\hline Hawaii & 20,284 & 13.64 & 1.58 & 3.67 & 11,482 & 19.32 & 3.02 & 12.21 \\
\hline Idaho & 25,499 & 15.51 & -2.09 & 2.26 & 29,171 & 24.73 & 1.27 & 7.60 \\
\hline Illinois & 257,160 & 15.39 & -1.01 & 4.41 & 294,438 & 29.45 & -1.18 & 3.72 \\
\hline Indiana & 117,587 & 15.87 & 1.03 & 4.36 & 156,728 & 32.08 & -2.68 & 6.84 \\
\hline lowa & 20,544 & 9.82 & 1.79 & 3.53 & 39,487 & 19.45 & -6.94 & 0.17 \\
\hline Kansas & 31,835 & 12.14 & 1.26 & 3.28 & 56,483 & 24.18 & -1.00 & 5.85 \\
\hline Kentucky & 68,833 & 19.18 & -1.40 & 1.86 & 64,991 & 27.20 & -1.12 & 2.66 \\
\hline Louisiana & 116,037 & 23.04 & 0.48 & 3.42 & 107,479 & 34.08 & -1.59 & 2.55 \\
\hline Maine & 16,608 & 14.28 & 0.70 & 2.32 & 12,631 & 33.19 & 5.19 & 15.04 \\
\hline Maryland & 112,314 & 10.84 & 0.85 & 2.93 & 61,440 & 27.84 & -1.06 & 6.32 \\
\hline Massachusetts & 124,984 & 11.88 & 0.56 & 2.85 & 87,508 & 26.88 & -1.35 & 0.94 \\
\hline Michigan & 221,936 & 17.52 & -0.74 & 4.98 & 242,204 & 41.48 & 2.57 & 8.06 \\
\hline Minnesota & 67,317 & 10.01 & -0.78 & 1.66 & 65,553 & 22.69 & -0.90 & 2.95 \\
\hline Mississippi & 56,892 & 22.15 & 2.87 & 5.15 & 37,776 & 45.74 & 3.01 & 9.77 \\
\hline Missouri & 127,031 & 16.98 & 0.19 & 4.29 & 91,779 & 32.01 & 2.03 & 6.00 \\
\hline Montana & 3,255 & 11.98 & 2.03 & 0.57 & 10,958 & 22.96 & 0.30 & 5.97 \\
\hline Nebraska & 13,175 & 11.67 & 1.86 & 3.52 & 36,676 & 22.57 & -1.17 & 3.87 \\
\hline Nevada & 71,118 & 22.64 & 1.65 & 9.59 & 72,373 & 26.01 & 2.32 & 8.49 \\
\hline New Hampshire & 12,987 & 9.53 & -0.05 & 4.22 & 11,725 & 28.97 & 12.03 & 11.42 \\
\hline New Jersey & 238,714 & 13.03 & 0.82 & 3.46 & 71,512 & 40.67 & -1.37 & 11.30 \\
\hline New Mexico & 47,078 & 30.41 & -2.45 & 4.85 & 49,628 & 27.37 & 0.63 & 4.37 \\
\hline New York & 213,316 & 11.72 & -0.17 & 3.15 & 671,079 & 32.57 & 0.77 & 3.93 \\
\hline North Carolina & 188,972 & 21.31 & 0.39 & 7.23 & 199,662 & 27.04 & 0.51 & 4.84 \\
\hline North Dakota & 1,909 & 6.78 & 1.06 & -1.17 & 5,713 & 13.27 & -1.58 & -0.61 \\
\hline Ohio & 236,359 & 15.82 & -0.69 & 3.70 & 261,367 & 42.84 & 0.16 & 8.33 \\
\hline Oklahoma & 56,764 & 16.83 & -0.01 & 1.37 & 80,865 & 30.14 & 1.62 & 2.23 \\
\hline Oregon & 77,716 & 20.22 & 0.17 & 5.88 & 70,020 & 24.63 & -1.35 & 6.77 \\
\hline Pennsylvania & 221,761 & 13.00 & 0.52 & 3.01 & 227,347 & 38.61 & -1.63 & 4.01 \\
\hline Rhode Island & 23,890 & 16.64 & -0.61 & 3.76 & 17,745 & 25.18 & -5.73 & -1.28 \\
\hline South Carolina & 158,135 & 23.81 & -0.81 & 5.94 & 47,716 & 29.05 & -2.17 & 3.17 \\
\hline South Dakota & 2,982 & 7.56 & -1.48 & 1.63 & 10,350 & 18.63 & 2.63 & 2.64 \\
\hline Tennessee & 98,865 & 16.52 & 0.09 & 1.54 & 177,384 & 35.11 & 0.02 & 5.14 \\
\hline Texas & 608,245 & 20.11 & -1.53 & 2.95 & 957,928 & 30.57 & -0.29 & 2.53 \\
\hline Utah & 79,326 & 12.72 & 0.20 & 4.66 & 39,362 & 25.97 & -1.42 & 7.93 \\
\hline Vermont & 3,106 & 9.25 & -2.79 & 2.41 & N/A & $\mathrm{N} / \mathrm{A}$ & $N / A$ & $N / A$ \\
\hline Virginia & 122,500 & 10.51 & -0.32 & 1.59 & 101,891 & 23.12 & 1.91 & 3.58 \\
\hline Washington & 129,916 & 14.57 & -1.07 & 2.28 & 114,771 & 23.44 & 1.98 & 4.70 \\
\hline West Virginia & 32,143 & 18.99 & -3.07 & 1.00 & 13,459 & 31.97 & -1.94 & 3.38 \\
\hline Wisconsin & 58,978 & 10.02 & -0.68 & 1.98 & 120,124 & 31.93 & 1.83 & 6.99 \\
\hline Wyoming & $N / A$ & $\mathrm{~N} / \mathrm{A}$ & N/A & $N / A$ & 5,249 & 19.83 & 7.11 & 12.86 \\
\hline
\end{tabular}

would be obtained using rounded figures. Bold font indicates a statistically significant change $(p<0.05)$. 
TABLE 2. YOUNG CHILD POVERTY BY PLACE TYPE AND REGION IN 2012

\begin{tabular}{|c|c|c|c|c|c|c|c|c|}
\hline & \multicolumn{4}{|c|}{ ALL PLACE TYPES } & \multicolumn{4}{|c|}{ RURAL } \\
\hline & $\begin{array}{l}\text { Number } \\
\text { Below } \\
\text { Poverty }\end{array}$ & $\begin{array}{l}\text { Percent } \\
\text { Below } \\
\text { Poverty }\end{array}$ & $\begin{array}{l}\text { Percentage } \\
\text { Point Change } \\
\text { Since } 2011\end{array}$ & $\begin{array}{l}\text { Percentage } \\
\text { Point Change } \\
\text { Since } 2007\end{array}$ & $\begin{array}{l}\text { Number } \\
\text { Below } \\
\text { Poverty }\end{array}$ & $\begin{array}{l}\text { Percent } \\
\text { Below } \\
\text { Poverty }\end{array}$ & $\begin{array}{l}\text { Percentage } \\
\text { Point Change } \\
\text { Since } 2011\end{array}$ & $\begin{array}{l}\text { Percentage } \\
\text { Point Change } \\
\text { Since } 2007\end{array}$ \\
\hline United States & $6,052,083$ & 25.51 & -0.07 & 4.97 & $1,077,356$ & 30.09 & -0.72 & 4.39 \\
\hline Northeast & 826,374 & 21.80 & 0.05 & 4.22 & 80,619 & 24.62 & 0.46 & 5.75 \\
\hline Midwest & $1,229,088$ & 24.51 & -0.69 & 5.19 & 272,179 & 25.28 & -1.29 & 4.23 \\
\hline South & $2,560,830$ & 28.21 & -0.21 & 4.50 & 575,206 & 35.23 & -0.44 & 4.17 \\
\hline West & $1,435,791$ & 24.56 & 0.60 & 5.90 & 149,352 & 27.51 & -1.15 & 4.72 \\
\hline
\end{tabular}

Note: Percentage point changes are based on unrounded poverty percentages and may differ slightly from those that

\section{End notes}

1. Although some states/places appear to have percentage point increases in poverty, those not in bold are not statistically distinguishable from zero.

2. See annual state ranking tables available at http://datacenter.kidscount.org.

3. Carsey Institute analysis of ACS data; available upon request.

4. Jeanne Brooks-Gunn, Greg J. Duncan, and Nancy Maritato, "Poor Families, Poor Outcomes: The Well-Being of Children and Youth," Chapter 1 in Consequences of Growing Up Poor, edited by Greg J. Duncan and Jeanne Brooks-Gunn (New York: Russell Sage Foundation, 1997). See also "Children at Risk: Consequences for School Readiness and Beyond," Rand Labor and Population Research Brief, retrieved from www.rand.org/content/dam/rand/ pubs/research_briefs/2005/RAND_RB9144.pdf (2005).

5. Children's Health Watch, “Federal Programs that Protect Young Children's Health,” Policy Action Brief (Boston, MA: Children's Health Watch, 2011).

6. Chuck Marr, Jimmy Charite, and Chye-Ching Huang, "Earned Income Tax Credit Promotes Work, Encourages Children's Success at School, Research Finds," (Washington, DC: Center on Budget and Policy Priorities, 2013).

7. See U.S. Census Bureau's American FactFinder, available at http://factfinder2.census.gov/ faces/nav/jsf/pages/index.xhtml.

8. Ibid.

9. Ibid. 
TABLE 2. YOUNG CHILD POVERTY BY PLACE TYPE AND REGION IN 2012, CONTINUED

\begin{tabular}{|c|c|c|c|c|c|c|c|c|}
\hline & \multicolumn{4}{|c|}{ SUBURBAN } & \multicolumn{4}{|c|}{ CENTRAL CITY } \\
\hline & $\begin{array}{l}\text { Number } \\
\text { Below } \\
\text { Poverty }\end{array}$ & $\begin{array}{l}\text { Percent } \\
\text { Below } \\
\text { Poverty }\end{array}$ & $\begin{array}{r}\text { Percentage } \\
\text { Point Change } \\
\text { Since } 2010\end{array}$ & $\begin{array}{r}\text { Percentage } \\
\text { Point Change } \\
\text { Since } 2007\end{array}$ & $\begin{array}{l}\text { Number } \\
\text { Below } \\
\text { Poverty }\end{array}$ & $\begin{array}{l}\text { Percent } \\
\text { Below } \\
\text { Poverty }\end{array}$ & $\begin{array}{r}\text { Percentage } \\
\text { Point Change } \\
\text { Since } 2011\end{array}$ & $\begin{array}{r}\text { Percentage } \\
\text { Point Change } \\
\text { Since } 2007\end{array}$ \\
\hline United States & $2,340,951$ & 19.79 & 0.10 & 4.99 & $2,633,776$ & 31.65 & 4.85 & -0.10 \\
\hline Northeast & 324,523 & 14.72 & 0.07 & 4.25 & 421,232 & 33.46 & 3.52 & -0.40 \\
\hline Midwest & 415,833 & 17.50 & -0.41 & 4.60 & 541,076 & 34.65 & 6.05 & -0.87 \\
\hline South & $1,003,197$ & 22.42 & 0.03 & 4.97 & 982,427 & 33.07 & 3.70 & -0.50 \\
\hline West & 597,398 & 21.54 & 0.62 & 5.85 & 689,041 & 27.24 & 6.03 & 0.95 \\
\hline
\end{tabular}

would be obtained using rounded figures. Bold font indicates a statistically significant change $(p<0.05)$.

\section{About the Authors}

Beth Mattingly is director of research on vulnerable families at the Carsey Institute and a research assistant professor of sociology at the University of New Hampshire (beth.mattingly@unh.edu).

Jess Carson is a vulnerable families research scientist at the Carsey Institute (jessica. carson@unh.edu).

Andrew Schaefer is a doctoral student in sociology at the University of New Hampshire and a research assistant at the Carsey Institute (andrew.schaefer@unh.edu).

\section{A cknowledgments}

The authors thank Jennifer Clayton, Barbara Cook, Curt Grimm, Ken Johnson, Laurel Lloyd, Luke Rogers, Amy Sterndale, and Barbara Wauchope at the Carsey Institute for their assistance, comments, and suggestions. 


\section{$\triangle$ UNIVERSITY - of NEW HAMPSHIRE}

\section{CARSEY}

Building knowledge for families and communities

The Carsey Institute conducts policy research on vulnerable children, youth, and families and on sustainable community development. We give policy makers and practitioners timely, independent resources to effect change in their communities.

This work was supported by the Annie E. Casey Foundation, the W. K. Kellogg Foundation, and anonymous donors.

Huddleston Hall

73 Main Street

Durham, NH 03824

$(603) 862-2821$

www.carseyinstitute.unh.edu 